\title{
In Detail; Research Reviews; Of New Interest
}

\author{
Nancy L. Segal, \\ Department of Psychology, California State University, Fullerton, California, USA
}

\begin{abstract}
T
wo families with twins - one in which adult DZ twins continue to face the risk of frontotemporal dementia, and the other in which a mother copes with brittle bones in a newborn infant MZ pair — are featured. The nature and progress of the disorders are reviewed and personal perspectives from the twins and other family members are variously provided. Next, four current twin studies are briefly summarized. Topics include sex differences in social support systems, new reproductive technologies, comparative twin-singleton frequencies of placenta previa, and the nature and complications of heterotopic pregnancies. This article concludes with a brief sampling of human interest stories involving twins: a different looking DZ pair, newborn sextuplets, conjoined twins and opposite-sex twin athletes.
\end{abstract}

\section{In Detail}

I hear of many medically important and emotionally challenging cases from friends, colleagues, parents and multiples. When these conditions involve twins, whether $\mathrm{MZ}$ or $\mathrm{DZ}$, unique aspects inevitably arise. This past year, I was put in contact with two unusual families whose stories span a wide range of exceptional circumstances, including risk of dementia, limitations of available medical information and difficulties coping with disabled twins. Members of these families graciously shared their experiences in the hope that their observations may benefit readers of Twin Research. There is little doubt that their goals will be fulfilled - the information they provided is scientifically enlightening and personally affecting.

\section{DZ Twin-Sibling Discussion of Frontotemporal Dementia (FTD)}

Frontotemporal Dementia with Parkinsonism has been linked to Chromosome 17 (FTDP-17); however, the precise gene involved has not yet been identified. At the center of the present case are three sisters, 42-yearold DZ female twins, Carrie and Colleen, and their 45-year-old sister, Monica. (Names and other identifying information have been altered to preserve confidentiality.) Their mother suffered from FTD which, according to Monica, was first expressed in the form of a shift from her being even tempered to easily angered and uncharacteristically depressed. This change became apparent when their mother was in her early to mid-40s. Given FTD's early age of onset and the fact that the twins and their sister are approaching/are within the period of risk, it seemed important to record their responses and recollections. I suspect that variations among their views partly reflect their geneticallybased differences in personality and temperament. It is also clear that the twins' outlook was partly affected by their being $\mathrm{DZ}$ twins, rather than MZ twins. Some background information on this severely debilitating disorder is first provided (Mann, 2001).

Frontotemporal dementia (FTD) was originally called Pick's Disease, named after the German physician
Arnold Pick who had conducted research in this area. The decision to name the disease after Pick was proposed in 1923 by Alois Alzheimer.

FTD affects approximately $1 / 5,000$ people. It usually strikes people in their mid-40s, most often before they reach 65 years of age, but it sometimes occurs in people in their 20s and 30s. The typical duration of the disorder is 10 to 15 years. Behavioral symptoms include disturbance and change in personality, including difficulties in concentration and alteration in attitudes toward others. Failure to understand word meanings and/or to recognize faces of familiar people may occur.

The genetic underpinning of FTD is a mutation located on chromosome 17. The normal form of the gene manufactures tau, a protein essential to proper nerve cell function. In the absence of tau, individuals show brain tangles characteristic of dementia. However, only half of all FTD patients show these

Address for Correspondence: Nancy L. Segal, Department of Psychology, California State University, Fullerton, CA 92834 USA.Email:nsegal@fullerton.ed 
brain changes, suggesting alternative inherited forms of the disorder.

Carrie, one of the DZ twins, informed me that a test is now available to identify people with and without the chromosome 17 mutation, but it has not been approved for general use. (She learned this from a researcher associated with a research project in which her family is now enrolled. The study is being conducted at Duke University's Bryan Alzheimer's Disease Research Center.) Perhaps researchers worry that younger people carrying the defective gene will experience severe emotional upset if they learn that they carry the mutated allele - apparently, anyone carrying the gene expresses the condition at some point in time.

\section{The Three Sisters}

Several of the sisters referred to their family's disorder as "the Big A". They were aware of it since junior high school since it "decimated" their mother's family. According to one sister, "In our family when you reach your fiftieth birthday, and you know it, you celebrate!"

I gathered information from each of the three sisters via a series of telephone calls between January and March, 2003. They expressed collective concerns, as well as individual perspectives, and all were illuminating. I will begin with comments from the DZ twins, Carrie and Colleen. Their classification as $\mathrm{DZ}$ was based upon differences in hair color and texture (Carrie: dark brown and coarse; Colleen: light brown and fine), as well as eye color (Carrie: brown; Colleen: hazel).

\section{Carrie}

My initial contact was with Carrie, a married mother of two and a very active and successful speaker and trainer. Carrie traced the transmission of FTD in her mother's family tree: affected individuals included her maternal grandmother and probably several of her grandmother's siblings. Among individuals in the next generation who were affected were her mother, her mother's older sister and an older brother. One sibling had passed away prior to the age of onset and two sisters, ages 57 and 68, are alive and functioning well - they have generally passed through the period of risk. The twins have a number of cousins, three of whom are daughters of their mother's affected older sister - the middle daughter expressed the disease at age 48 and currently resides in a nursing home.

Carrie perceives herself as the "emotional twin" and her sister as the "practical twin." The possibility that she may carry the lethal gene plays out in every aspect of her life, including relationships and risks. It is a game against time - with 6 or 7 years left to pass through, life must be lived to the fullest. Some people might take the position that nothing matters, but Carrie has taken the opposite view. She focuses on maintaining good health through exercise and meditation. To some degree she sees the disease process as a "blessing," namely an opportunity for personal growth. She feels "it is not fair" that the diagnostic test is unavailable, although she is not sure others in her extended family would be able to "weather bad news". However, given that she cannot know the truth for several years, she is determined to live life as though she does have the dreaded gene, which means living life to the fullest.

Carrie has two adopted children. The decision to adopt was not prompted by fear of passing on the disease gene to biological children, but was a consequence of unexplained infertility. Should she become ill her children would be younger than she was when her own mother became affected. This is clearly a worry. Another key concern is the fact that she is unaware of how her own mother felt when her symptoms first appeared. This information could be invaluable to people in her situation and to their families.

\section{Colleen}

I spoke with Carrie's twin sister, Colleen, one month later, focusing on experiential aspects of twinship and her thoughts as she moves through this crucial period of risk. When I asked her what it was like to be a twin, she replied, "What is it like not to be a twin?" (As a DZ twin myself, I could have guessed this answer.) The twins were close while growing up, and somewhat competitive, but less competitive than twins in three other DZ pairs she knew. Competition was most pronounced over school grades, although each twin excelled in different areas.
Carrie was also more popular, a "part of the in-crowd".

How does the possibility of FTD affect her life? She said it does not. She sees no evidence that she has the disorder. "We [my twin and I] deal with it differently. Carrie worries that she has it, I don't feel that I have it at all". But she believes Carrie wouldn't "dwell" on it unless there was some reason to do so, unless there was some sign. She is aware that if her sister did have FTD, she herself would not be at similar risk because they are not MZ twins. "If we were identical twins and she [Carrie] thought she might have it, then it would put more pressure on me". Like her twin sister, Colleen regrets the lost opportunity to understand her mother's early experience with the disease. She only knows that her mother would say, "I am losing my mind" and that "the Big A" was responsible.

Colleen emphasized how trying she found the experience of witnessing her mother's progressive mental deterioration. "She had seen her siblings go through it so in the early stages it must have been so difficult for her. It was easier for our family when my mother was too ill to understand".

\section{Monica}

I asked Monica to comment on her sisters' relationship, on her own special role as their older sister and on her thoughts about the disease risk she is facing. She provided another take on their twinship and another view of life lived with the possibility of FTD.

According to Monica, when the twins were very young they "banded together". "My mother told me that they were my babies when they were born. I thought that was remarkable and I regarded them as my possessions. I paraded them around. When they were old enough to walk and talk I became jealous of them because I no longer needed to introduce them". Monica is aware of the twins' different personalities, but also of their caring and loyalty toward each other, even while being occasionally judgmental. She is also aware that all three sisters cope very differently with the family problem. Here, she referred to Carrie's sensitivity and Colleen's independence in managing their feelings. When asked how she copes, she explained that each day she is challenged to learn 
new things and so far has sensed no change in her mental capacities. She finds it amusing when people comment on her excellent memory. She claims to feel no fear — but her sisters suspect she is in denial.

Monica is aware of the definitive test for FTD, even though it is not being made available. Would she take it if it were? "No" she said. "I would only have it if I were concerned about symptoms". But knowing might help her to deal with her feelings by reaching out to others. She recalled that her own mother failed to express her own fears.

She added that some people felt that her mother's behavior was controllable and that she could have acted more responsibly. Of course, this was not true and Monica is grateful that more is becoming known about dementia. "You can still live with dignity. I have great memories of my mother".

\section{Comment}

There are currently no twin studies of FTD. According to Carrie, only eight or nine families are currently enrolled in the Duke University project in which her family is participating. A twin study could yield important sources of individual variation. If, for example, MZ co-twins differed in symptoms and/or severity, it would be of interest to determine which factors might be responsible.

Additional information about ongoing dementia-related research at Duke University is available at http:// adrc.mc.duke.edu/research.htm and at http://chg.duke.edu/programs. html

\section{A Mother's Reflections on Brittle Bones in MZ Infant Twins}

Osteogenesis imperfecta (OI) is characterized by bones that fracture easily for no obvious reason. OI is a geneticallybased condition and the most common disease-based cause of childhood fractures (although it can cause fractures in adulthood). It results from gene abnormalities associated with the production of collagen, a protein vital to maintaining bone strength. Approximately 20,000 to 50,000 people in the United States are affected by one of the four forms of the condition (Dundee
University, 2000; Osteogenesis Imperfecta Foundation, 1999).

Brittle bone syndrome (BBS) is a special form of OI that has attracted medical attention during the last two decades. Bone fractures occur during the first year of life, often during the first 6 months. The condition is considered temporary as it generally disappears after children reach their first birthday. The cause of the disease is unknown, but multiple birth and prematurity are known risk factors. Womersley (2000) reviewed a British case in which parents of affected infant male twins had been accused of child abuse. A judge eventually pardoned the parents, based on expert testimony, although one of the three consultant pediatric radiologists claimed that the condition was non-existent.

The case I will describe concerns infant MZ female twins for whom a diagnosis of brittle bone syndrome was initially suspected, but was since disconfirmed. This case reveals the limits of medical knowledge in certain areas, and highlights the unique experiences of parents and twins, some heart-warming, others heart-breaking. Identifying information has been altered to mask the identity of the family.

Ilene C., a single mother aged 37, delivered $\mathrm{MZ}$ twin girls in January, 2002. Her pregnancy was complicated by an incompetent cervix, and she went into labor at 20 weeks. Following three months of bed rest, Ilene delivered $\mathrm{MZ}$ twin girls by cesarean section at 32 weeks. Their zygosity was established by DNA analysis. The twins were named Susan and Rachel. Chest X-rays done on the twins soon after birth indicated broken ribs and multiple fractures. Further examination revealed that their bones were quite thin, consistent with Brittle Bone syndrome. As indicated above, some children show this condition during the first year of life, after which it disappears; hence, the name Temporary Brittle Bone syndrome (TBBS). However, Susan and Rachel's symptoms did not fit the pattern typical of TBBS.

The twins lived with their mother for their first 6 months, during which time they were fracture-free. (Most TBBS children experience fractures during the first year.) They were then moved to a special care facility because other physical complications made them difficult to care for. Each showed a dysmorphic (misshapen) face and microcephaly (congenitally small head and brain). Susan, the firstborn twin, also underwent a brain hemorrhage so it is likely she will be affected with cerebral palsy. A geneticist performed a skin biopsy on one of the twins to confirm Brittle Bone syndrome, but the result proved negative. According to Ilene, this test is approximately $85 \%$ accurate; however, Brittle Bone syndrome was an unlikely diagnosis because the twins had not had fractures since birth. At present their condition lacks a definitive diagnosis.

I spoke with Ilene when the twins were 14 months old. Rachel was still in the special care facility and would be released to her mother's care 2 weeks later. Two days earlier, Susan has been adopted by a family from another state. The decision to separate the twins did not come easily and Ilene's sadness and uncertainty over this decision were evident in her comments that followed. "I gave up my child 2 days ago. I am not thinking straight". She explained that adoption of one or both babies was not part of her initial plan. Knowing that her twins had Brittle Bone syndrome was difficult to accept, but such children are of normal intelligence and she was prepared to raise them. But signs of microcephaly indicated that they would be severely mentally retarded; as such, they entered the special care facility in October, 2002. The difficulties involved in raising these two children made Ilene think seriously about relinquishing them for adoption.

However, Rachel began to improve, showing signs of normal behavioral and motor development despite her unusual facial features and small head. This, coupled with the attachment Ilene felt toward the child during the 6 months she cared for her at home, made her decide to resume care herself. Unfortunately, Susan's conditioned worsened to the point at which Ilene felt she could not care for her adequately, so sought assistance from an adoption agency and adoption was recently arranged. The adoption will be open, allowing four visits each year. Everyone involved respects the twin relationship, so are in favor of the twins maintaining social contact. Of course, this may not be easy given the 
considerable physical distance between the families.

How does Ilene feel? She is nervous about Rachel coming home and caring for a normally developing child whose appearance is not normal. She struggles with the thought that she separated the twins because of the general consensus that twins gain immeasurably from one another's companionship. She wonders whether both girls might have fared better had they been kept together after all, they share the same physical abnormalities and may have been comforted by being with someone who would completely understand them. But Ilene admits that she could not handle two babies and must accept this decision which is now final.

\section{Comment}

It is impossible to predict each twin's physical and emotional outcomes in this case. Most people agree that twins should be kept together, so when deliberate decisions keep them apart it may be difficult to understand. However, each family's situation is unique and must be evaluated as such. Had Rachel and Susan remained together, it is conceivable that their physical and mental health differences would have prevented them from forming a meaningful relationship. It is also possible that close physical association would have proven a burden to Rachel (the healthier twin) who may have forfeited opportunities and experiences for the benefit of her co-twin (see Bryan, 1992; Segal, 2000). I once provided legal assistance for a case involving separate versus common placement of DZ twin children, one of whom was severely handicapped (Segal, 2000). While I strongly believe twins should stay together whenever possible, this situation proved exceptional. Given that the healthy twin was often forced to sacrifice scheduled events and other activities when her sister's health status required it, and because unfair (and unrealistic) co-twin comparisons were sometimes made by others, I recommended separate rearing.

Another possible outcome is that a warm relationship may have resulted from the twins' staying together; in fact, many such examples have been documented (New York Times, 1999). Certainly, excellent care is essential for children with severe handicaps such as Susan's - without it, a close relationship between the twins would have been unlikely. Parents like Ilene generally have their children's best interests in mind when making important decisions, even those that are painful.

The greater frequency of brittle bones in twins, coupled with its rarity, means that collaborative efforts are required for research targeted toward refining diagostic criteria and developing effective cures. Susan and Rachel's condition is currently without a name, given that Brittle Bone syndrome has been ruled out - physicians refer to their disease as "something genetic," but have no further clues. A review of family histories has offered no promising leads. Medical researchers whose practices include twins with Brittle Bone syndrome and other forms of OI will, hopefully, pool existing cases in the search for answers.

\section{Research Reviews}

\section{Twins' Social Support Systems}

There is interest in understanding the nature of individuals' social support systems, given evidence that social support decreases risk of psychiatric illness. A recent twin study examined sex differences in social support dimensions with reference to genetic and environmental underpinnings (Agrawal, Jacobson, Prescott, \& Kendler, 2002). Twin participants were drawn from the Virginia Twin Registry, now part of the Mid-Atlantic Twin Registry. They included MZ, DZ same-sex and DZ opposite-sex twin pairs, as well as several triplet sets and one quadruplet set. Social support was assessed by a 16-item scale. Factor analysis yielded six dimensions: friend support, relative support, friend prob- lems, relative problems, confidants and social integration.

Genetic influence was detected for all social support dimensions, with the exception of relative support in males. Shared environmental effects were observed for relative support and relative problems among both males and females. Sex differences in mean ratings were found for all dimensions except for confidants and relative support, with females exceeding males. The investigators concluded that despite mean differences in most social support dimensions between males and females, sources of variation differed for some aspects of relative support and confidants only. They also noted several limitations of the study, such as exclusive reliance on self-report measures and the use of an ethnically homogeneous (Caucasian) twin sample.

\section{DZ Twins From Thawed Human Blastocycts}

Considerable debate has surrounded the optimal time for freezing and preservation of unimplanted embryos (cryostorage). Some investigators have favored the blastocyst stage over earlier stages, yet there is risk posed by the possible loss of embryos that do not withstand lengthy intervals in in vitro culture.

A recent report of the births of infant twins following transfer of a fresh, seven-day blastocyst suggested that cryopreservation of 6- and 7-day-old embryos could prove successful (Sagoskin, Han, Graham, Levy, Stillman \& Tucker, 2002). This 2002 case provided the impetus for the present one, namely the first delivery of twins following in vitro fertilization (IVF) of one 6-day-old and two 7-day embryos, cryo-preservation and intrauterine 
transfer of embryos via intracytoplasmic sperm injection (ICSI) approximately one month later (Sills et al., 2002).

\section{Placentia Previa in Twins and Singletons}

Placentia previa is a pregnancy complication in which the placenta becomes wholly, or partially, located in the lower portion of the uterus. When it becomes stretched during the later part of pregnancy and when the cervix becomes stretched before or during labor, placental separation and hemorrhage can occur. Especially severe cases are those in which the placenta is located before the presenting part of the fetus, mandating cesarean delivery (Bantam Medical Dictionary, 1994). Placentia previa has been estimated to occur in $2 / 1,000$ to $6 / 1,000$ pregnancies and has been held responsible for $5 \%$ of perinatal deaths (see Ananth, Demissie, Smulian, \& Vintzileos, 2003).

A comparative analysis of the frequency of placenta previa and associated risk factors in twin and non-twin births was recently undertaken by investigators at the University of Medicine and Dentistry of New Jersey (Ananth et al., 2003). Reasons prompting the study were that placenta previa appeared to occur more often among twin than singleton births, and that risk factors were uncertain. Data were drawn from the United States natality files (1989-1998), compiled by the National Center for Heath Statistics. Cases were restricted to infants delivered by cesarean section. Medical life histories and demographic information were also available from these files.

The paper reports an extensive series of findings so only a selective sampling is provided here. Placenta previa was identified in 2.8/1,000 singleton births and in 3.9/1,000 twin births. It was shown to increase with maternal age and gravidity among both twin and non-twin mothers. The greatest risk occurred among women between the ages of 40 and 49 who had had five or more pregnancies; this was true regardless of whether they were carrying twins or singletons. Caucasian women were at greater risk than black women for placenta previa if they delivered twins, but no differences were detected if they delivered non-twins. Cigarette smoking as a risk factor for placenta previa was elevated among mothers carrying twins, relative to mothers carrying singletons.

The increased risk of placenta previa among twin births is a significant concern. Ananth et al. speculated that it might be linked to postponement of pregnancy and increased use of artificial reproductive technologies which increase the probability of multiple birth. Specifically, large placentas resulting from twinning could "encroach" upon the cervical area. Careful monitoring of multiple birth pregnancies with a view toward the possible presence of placenta previa was urged.

\section{Heterotopic Twin Pregnancy}

Heterotopic pregnancy is the rare cooccurrence of an intrauterine and an ectopic pregnancy. (An ectopic pregnancy involves fetal development at a site other than the uterus, the most common being one of the Fallopian tubes.) These unusual conceptions have been on the rise in recent years, given that risk factors include the use of fertility drugs or other artificial reproductive procedures.
A naturally occurring heterotopic pregnancy was recently reported by Dr. E.A. Aneziokoro, from the Department of Gynecology and Obstetrics at the Ebonyi State University Teaching Hospital, in Nigeria (Aneziokoro, 2002). An ectopic pregnancy was detected in a 24-year-old pregnant woman who had experienced weakness, spotting and abdominal pain. She had been pregnant for 8 weeks. A ruptured tubal pregnancy was detected in her left Fallopian tube, necessitating salpingectomy (surgical cutting or removal of the tube). However, recurrence of weakness and abdominal tenderness caused her to seek medical attention one month later. At this time, a normal intrauterine pregnancy was diagnosed. She maintained the pregnancy until term before delivering healthy opposite-sex twins. The female and male twin infants were nicknamed Testimony and Miracle, respectively, by their parents.

Ectopic pregnancies may be lifethreatening, but early detection is not always possible. Furthermore, only twothirds of heterotopic pregnancies eventuate in normal births; approximately one-third are aborted. (Interestingly, at the time the report was published, the mother was 15 weeks pregnant with another child.) In addition to artificial reproductive technologies, the author cites the following as risk factors for ectopic pregnancies: pelvic inflammatory disease, sexually transmitted diseases, intrauterine contraceptive devices, previous abdominal surgery, previous ectopic pregnacy, progestin-only oral contraceptives, delayed marriages and tubal surgery.

\section{Of New Interest}

\section{Ethnically "Different" Twins}

Selected twin pairs sometimes suggest interesting new projects because of their unusual behavioral, physical or life history features. In Fall, 2002, I recruited several twin pairs from a developmental psychology class for par- ticipation in studies of cooperation and competition. An Hispanic female student in my class offered to bring her DZ twin sister to my laboratory. When the pair arrived, my assistants and I were surprised and intrigued by these ethnically "different" twins - my student's co-twin appeared to be Caucasian! The twins explained that their father is Hispanic and their mother is Caucasian, and that the two have always looked extremely different.

Most interesting was the news that the twins had experienced very different levels of discrimination. It seems that the Hispanic-looking twin had, 
from time to time, been subjected to unkind comments, based on her appearance, while her co-twin had never encountered such prejudice. This poses an unusual situation, namely the idea that twin sisters who were raised together and share the same biological background can have such contrasting life experiences. Studying a sample of ethnically "diverse" DZ twin pairs might yield fresh insights into the bases of discrimination and individuals' responses to it.

Photographs of the twins appear in Figure 1.
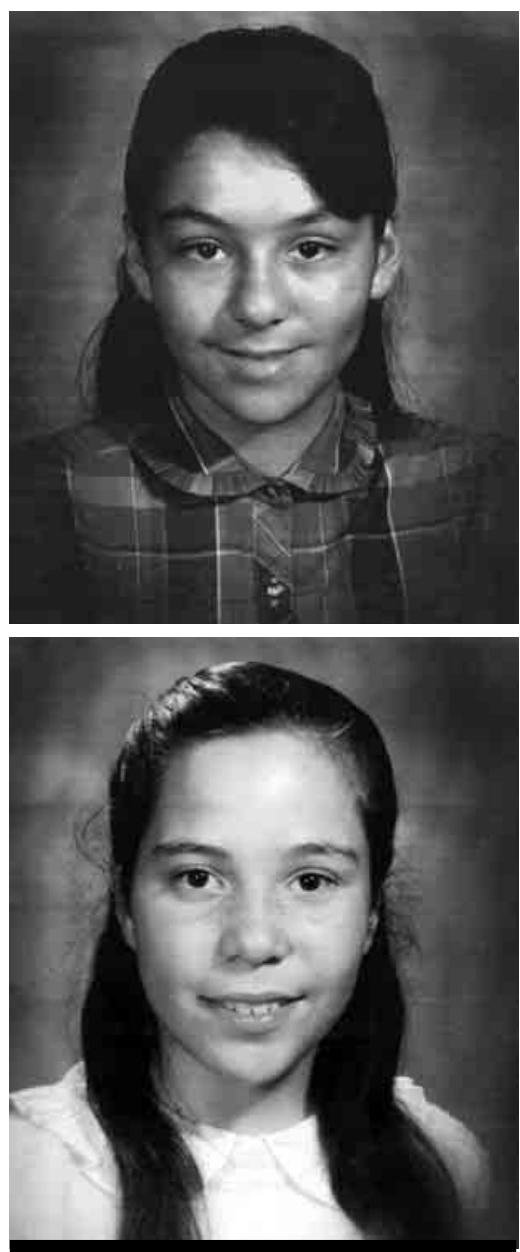

Figure 1

"Ethnically different" DZ twins at age 11 years. Photo courtesy of the twins.

\section{Sextuplets in Pittsburgh}

Fertility drugs were responsible for the recent birth of sextuplets to a Pittsburgh, Pennsylvania mother (New York Times, 2003). The infants included three male and three female infants, born 12 weeks premature. In all six cases, breathing was assisted by the use of ventilators. The prognosis for the sextuplets is considered favorable.

Advances in medical technology and pregnancy management is improving the physical outcomes of higher order multiples, yet persistent health problems and developmental delays are well known (Emanuel, 1999). Couples need to be fully apprised of the possible multiple birth consequences of fertility drugs and other reproductive treatments.

\section{Conjoined Twins}

High medical costs have delayed, and may possibly cancel, planned surgical separation of conjoined Egyptian twins, Ahmed and Mohamed (New York Times, 2003). The twins, now 22 months old, were brought to Dallas, Texas for the operation by the World Craniofacial Foundation. They are craniopagus twins, joined at the top of the head (see www.egyptiantwins. com). An additional $\$ 125,000$ is needed to meet the operation's total cost of $\$ 2,000,000$. Surgical separation was scheduled to take place at the Children's Medical Center of Dallas; however, concern that the enormous expense would divert medical resources from North Texas children has prevented physicians from moving forward.

The twins' outcome remained uncertain, especially since they are now older than most conjoined twins who undergo separation. Surgery tends to be more successful when twins are older (between 6 to 12 months of age; O'Neill, Holcomb III, Schnaufer, et al., 1988), yet Ahmed and Mohamed are considerably older. Little is known about the emotional consequences of such delayed separation in conjoined twins. However, on April 28, physicians at Dallas
Children's Medical Center took intial surgical steps toward separting the twins (NBC News, 2003).

\section{Opposite-sex Twin Athletes}

We often hear about the matched athletic abilities of $M Z$ twins, such as Olympic skiers, Phil and Steve Mahre, Olympic rowers, Betsy and Mary McCagg and Olympic synchronized swimmers, Karen and Sarah Josephson (Segal, 2000). However, DZ twins are less likely to perform at the same ability level, so when they do, this generates considerable interest. High school seniors, Colleen and Kieran Carney, are male-female twins from Yorktown, New York who show exceptional skills in lacrosse (Slater, 2003). Their older brother, Sean, is also an outstanding player. The twins and siblings share half their genes (on average), as well as their family environments, so it is impossible to pinpoint the source of their athletic prowess - most likely both are involved. However, Sean's sports interests do seem partly responsible for those of his younger twin siblings since he often took them to practices and games.

The most intriguing aspects of these athletic twins are their extraordinary skills and close relationship. Kieran has been described as "one of the premier players in Section 1, if not the state." Colleen received section honors and an All-American honorable mention. The twins play the same position on their respective teams. They give each other advice on how to play - DZ twins tend to be more competitive than $\mathrm{MZ}$ twins, yet these twins do not play against each other, perhaps facilitating an amicable relationship. Lacrosse also offers them a meaningful shared interest. One of the twins commented that "it" [lacrosse] gives them something to talk about, although they get along fine regardless. a

\section{Acknowledgment}

This work was supported, in part, by NIMH Grant I ROI MH63351 and a Senior Faculty Research Award for CSUF Faculty. 


\section{References}

Agrawal,A., Jacobson, K. C., Prescott, C.A., \& Kendler, K. S. (2002). A twin study of sex differences in social support. Psychological Medicine, 32, I I55-I I 64.

Ananth, C. V., Demissie, K., Smulian, J. C., \& Vintzileos, A. M. (2003). Placenta previa in singleton and twin births in the United States, 1989-1998: A comparison of risk factor profiles and associated conditions. American Journal of Obstetrics and Gynecology, 188, 275-28I.

Aneziokoro, E. A. (2002). Heterotopic pregnancy with live twins. African Journal of Reproductive Health, 6, I I7-II9.

Bantam Medical Dictionary. (1994). New York: Bantam Books.

Bryan, E. M. (1992). Twins and multiple births. New York: Little Brown.

Dundee University (2000). Brittle bone disease - osteogenesis imperfecta Retrieved from http://www.dundee.ac.uk/ medicine/taynendoweb/images/brittle_bo ne_disease.htm

Emanual, E. (1999). Eight is too many. New Republic, 8, I0-II.

Mann, D. (200I). Frontotempral dementia Fact sheet. Manchester, England: North West Centre, Personal Social Sciences Research Unit.

NBC News (2003). Procedure prepares conjoined twins for seperation. (www. msnbc.com/local/kxas/a l 595778.asp).

New York Times (mid-Atlantic, 2 I March, 2003), Pennsylvania: Sextuplets born. p.A-16.

New York Times (Southwest, 2 April, 2003), Texas: Surgery for joined twins in doubt. p.A-18.

New York Times (Long Island, 7 June, 1999). An identical twin's dance toward independence. p. B-8.

O’Neill, J. A., Holcomb III, G. W., Schnaufer, L., Templeton, J. M., Bishop, H. C., Ross
III,A. J., et al. (1988). Surgical experience with thirteen conjoined twins. Annals of Surgery, 208, 299-312.

Osteogenesis Imperfecta Foundation (1999). Fast facts on osteogenesis imperfecta. Retrieved from http://www.oif.org.

Segal, N. L. (2000). Entwined lives: Twins and what they tell us about human behavior. NY: Plume.

Sills, E. S., Sweitzer, C. L., Morton, B. S., Perloe, M., Kaplan, C. R., \& Tucker, M. J. (2003). Dizygotic twin delivery following in vitro fertilization and transfer of thawed blastoysts cryopreserved at day 6 and 7. Fertility and Sterility, 79, 424-427.

Slater, C. (30 March, 2003). Twins score big on Lacrosse fields. New York Times, p.WC-2.

Womersley, T. (18 October, 2000). Brittle bones are being diagnosed as child abuse. Daily Telegraph. Retrieved from www.whale.to/m/sbs $26 . h t m l$. 